\title{
Bemerknngen zu dem von Schultze mitgetheilten Fall von „tuberculöser Iritis und Keratitis parenchymatosa".
}

Von

Dr. W. Zimmermann, Augenarzt in Stuttgart.

Aus äusseren Gründen komme ich erst jetzt dazu, zu der Schultze'schen Arbeit über "tuberculöse Iritis und Keratitis parenchymatosa " 1), einige Bemerkungen zu machen, um einer unrichtigen Deutung des anatomischen Befundes des seiner Zeit von mir publicirten Falles von Keratitis parenchymatosa tuberculosa ${ }^{2}$, auf welche sich Schultze zur Vertretung der von ihm angenommenen Genese der parenchymatösen Keratitis stützen zu können glaubt, entgegen zu treten. Ich halte es um so mehr für geboten, diese Auffassung meiner Befunde an dieser Stelle zu besprechen, als ich selbst mit durch die klinische und anatomische Untersuchung des erwähnten Falles gerade zu der von Schultze angefochtenen Ansicht über das Zustandekommen parenchymatöser Hornhauttrübungen gekommen bin. Es handelt sich darum, ob in Fällen von Keratitis parenchymatosa die Erkrankung der Hornhaut von der vorderen Kammer aus erfolgt, oder ob sie auf anderen Wegen, z. B. durch die Blutgefässe, resp. durch Erkrankungen der Gefässhaut eingeleitet wird.

Bekanntlich hat $v$. Hippel ${ }^{3}$ ) schon darauf hingewiesen, dass

1) Schultze, Archiv f. Augenheilk. Band XXXIII. S. 145.

2) Zimmermann, v. Graefe's Archiv f. Ophthalm. Bd. XLI. S. 215 .

s) v. Hippel, „Ueber Keratitis parenchymatosa“, v, Graefe's Archiv f. Ophthalm. XLII. S. 194 ff. 
Bemerkungen zu dem von Schultze mitgetheilten Fall etc. 259

die Schlussfolgerungen Mellinger's und Bärri's ${ }^{1}$ ), dass die menschliche Keratitis parenchymatosa eine primäre Erkrankung des Hornhautendothels sei, nicht zu Recht bestehen, weil bei den experimentellen Untersuchungen Bärri's stets eine traumatische oder chronische Läsion des Hornhautendothels voransging.

Trotzdem ist Schultze, und zwar wie er angiebt, auf Grund der anatomischen Befunde von Baumgarten, Meier, Zimmermann und im Hinblick auf seine eigenen Untersuchungen, zur Annahme geneigt, dass bei Erkrankungen constitutioneller Natur, wie Syphilis und Tubereulose durch Anwesenheit von Toxinen im Kammerwasser eine Ernährungsstörung des Endothels der Hornhaut und der Membrana Descemeti zu Stande kommen, so dass das pathologiseh veränderte Kammerwasser in die Grundsubstanz der Hornhaut eindringe und auf diese Weise parenchymatöse Trübung verursache. Weiter sagt Schultze am Schlusse seiner Abhandlung: "Gleichzeitig vorhandene Erkrankungen des Uvealtractus bei Keratitis parenchymatosa sind meiner Ansicht nach nicht die Ursache der Keratitis, denn sie werden oft vermisst, sondern nur ein häufig vorhandener Nebenbefund; bei $\mathrm{Er}$ krankungen der Iris und des Kanmerwinkels (Tuberkel, Syphilome) können dieselben das Kammerwasser wahrscheinlich stark mit Entzündungsproducten durehsetzen und somit die Entstehung der Keratitis hervorrufen."

Bevor ich auf die Frage der Entstehung der Keratitis parenchymatosa eingehe, möchte ich mich zunächst dagegen wenden, dass Schultze den von mir angenommenen Infectionsmodus für unrichtig hält; es handelt sich im Wesentlichen um zwei Stellen:

S. 149 heisst es: "Obgleich Zimmermann weder anamnestisch noch objectiv irgend welchem Anhaltspunkt für eine tuberculöse Erkrankung eines anderen Körpertheiles nachweisen konnte, nahm er doch an, dass das Auge nicht den primären Sitz der Tuberculose darstelle, sondern glaubt, dass eine secundäre Infection des Auges vorliege; einen Grund für diese Annahme führt er aber nicht an. Mir scheint in diesem Fall sehr wohl eine primäre tuberculöse Infection des Auges vorliegen zu können von der Conjunctiva aus in der oben erwähnten Art und Weise".

1) Bärri, Experimentelle Keratitis parenchymatosa, hervorgerufen durch Einwirkung auf das Endothel der Hornhaut. Inaug.Dissert. Basel 1895. 
Diesen Infectionsmodus denkt sich Schultze folgendermaassen: S. 146: "Warum soll nun nicht auch durch die Conjunctivalschleimhaut hindurch, eventuell nach einer unscheinbaren oberflächlichen Epithelwunde, eine Resorption von Tuberkelbacillen oder deren Sporen stattfinden können, die dann erst in der Gegend des Kammerwinkels an der Iriswurzel zur Entwicklung von Tuberkeln Veranlassung geben, während die kleine Wunde der Conjunctiva, falls überhaupt eine solche vorhanden war, spurlos verheilt ist? Unter den zahlreichen Bakterien, welche erwiesenermaassen permanent sich im Conjunctivalsacke befinden, sind gewiss auch zuweilen Tuberkelbacillen oder deren Sporen aus der Luft".

Wenn ich "trotz dęs negativen Ergebnisses der Anamnese und des objectiven Befundes" zu der Ansicht gelangte, dass es sich in dem in Rede stehenden Falle um eine endogene, also eine secundäre Infection und nicht um eine primäre Tuberculose des Auges handle, so habe ich allerdings an der von Schultze citirten Stelle der Arbeit (S. 231) für diese Ansicht keine Gründe mehr angeführt, da sich die Gründe, auf welche sich diese Annahme stützt, an anderen Stellen der Epikrise und bei Mittheilung des Befundes deutlich und klar ersichtlich finden.

Für eine ektogene Infection ergab weder der klinische Befund noch die anatomische Untersuchung einen Anhaltspunkt.

Es liegt mir ferne, zu behaupten, dass im normalen Bindehautsack nicht $\mathrm{ab}$ und zu auch lebensïähige Tuberkelbacillen angetroffen werden können; es wird ja vielleicht auch noch nachgewiesen werden, dass die Tuberkelbacillen dureh intactes Epithel hindurchwandern können und erst in tiefer gelegenen Organtheilen ilrr zerstörendes Werk beginnen! Aber vor der Hand stehen diese Annahmen in der Luft und entbehren vor Allem jeder Analogie. Wir wissen durch die experimentellen Arbeiten Valude's ${ }^{1}$, welcher schon früher darauf hinwies, dass die relative Seltenheit der Bindehauttuberculose wohl mit Wahrscheinlichkeit auf der baktericiden Eigenschaft der Thränenflüssigkeit beruhe, dass es ihm nicht gelungen ist, durch Impfung des Thränensackes mit einer Reincultur von Tuberkelbacillen eine Tuberculose der Thränensackschleimhaut zu erzeugen. Valude zieht aus seinen experimentellen Untersuchungen den Schluss, dass der natürliche Inhalt

1) Valude, Referirt in Münchener medicin. Wochensehrift, 35. Jahrg. S. 576. 
Bemerkungen zu dem von Schultze mitgetheilten Fall etc. 261

des Thränensackes den Tuberkelbacillus zerstöre und dessen Ansiedlung daselbst und in der Conjunctiva unmöglich mache: - Warum also in einem Falle, wie dem meinigen, einen derartig gesuchten Infectionsmodus annehmen an Stelle einer Infectionsart, wie sie vielfach beobachtet und auch durch anatomische Untersuchungen wissenschaftlich anerkannt wurde? Ich erinnere nur an die primären Tuberculosen der Gelenke und an oft lange Jahre latente Tuberculosen einzelner Bronchialdrüsen. Dass ich die Frage, ob eine Infection durch die Bindehaut (allerdings nicht durch die normale, wie Schultze annehmen will!) vorgelegen haben kann, reiflich geprüft, geht abgesehen von dem klinischen Befunde aus folgender Stelle, von weleher allerdings Schultze merkwürdiger Weise bei der Verwerthung meines Falles für seine Hypothese gar keine Notiz nimmt, zur Genüge hervor:

S. 233: „Besondere Beachtung verdient noch die Conjunctiva, welche in verschiedener Intensität von tuberculösen Heerden durchsetzt ist. Aus der Krankengeschichte ist zu entnehmen, dass der Anfang der Bindehauterkrankung in die auf die subconjunctivalen Injectionen folgende Zeit fällt; es ist ausdrücklich erwähnt, dass die Blutungen keine Neigung zur Resorption zeigten, und dass die Vernarbung eine ungewöhnliche war. Aus der mikroskopisehen Beschreibung geht hervor, dass eine Propagation der tuberculösen Heerde von der Sklera aus nach dem episkleralen Gewebe hin stattgefunden und dass an einer Stelle der in den episkleralen Lymphraum erfolgte Durchbruch eines Knötchens deutlich zu erkennen ist. Wir sind deshalb zu dem Schlusse berechtigt, dass sich die Verbreitung des tuberculösen Processes auf die Bindehaut an die subconjunctivalen Injectionen anschloss".

Also zu einer Zeit, in welcher bei bestehender Tuberculose der tiefer gelegenen Organe eine Läsion der Bindehaut gesetzt wurde, trat die Betheiligung der Bindehaut an dem tuberculösen Processe auf; dies ist klinisch und anatomisch sicher gestellt. Soll man andererseits rein willkïrlich, ohne irgend welche Stïtze an dem objectiven Befunde einfach annehmen, dass die Tuberkelbacillen, ohne Spuren zu hinterlassen, ihren Weg durch die Bindehaut genommen, sei es nun durch eine "unsichtbare" Epithelwunde, oder durch intactes Epithel?

Auch die Art, wie ich die endogene, secundäre Infection erklärte, ist genau angegeben.

S. 232 heisst es: "Hinsichtlich der Art der endogenen Infection und der zeitlichen Verbreitung des tuberculösen Processes 
ist zu bemerken, dass der Anfang der Erkrankuug, wie aus dem klinischen Berichte hervorgeht, an den äusseren Skleralbord, resp. in die Lederhaut zu verlegen ist. Unterstützt wird diese Annahme ferner durch den histologisehen Befund, welcher ausgedehnte tuberculöse Wucherungen in nächster Umgebung der vorderen Ciliargefässe erkennen lässt. Von hier aus fand zunächst eine Propagation in der Art der continuirlichen Fortsetzung auf den skleralbord und die Hornhaut statt.

$\mathrm{Ob}$ die sechs Monate nach Beginn der Erkrankung auf der medialen Seite auftretenden Trübungen auf eine neue Invasion durch die Blutgefässe zurückzuführen ist, oder ob die Infectionserreger in älnlicher Weise, wie wir dies für die Bindehaut annehmen müssen, auch von episkleralen Lymphraum aus in die Hornhant, resp. Lederhaut der anderen Seite ihren Eingang gefunden, entzieht sich der Beurtheilung".

Ich glaube somit annehmen zu dürfen, dass ich den Grund, warum ich hier eine Infection durch die Blutbahn, also eine secundäre Affection des Sehorganes als gegeben erachtete, deutlich genug hervorgehoben habe.

Für die Richtigkeit meiner Annahme spricht auch der weitere Krankheitsverlauf: Patientin erkrankte nämlich - ich verdanke diese Mittheilung der Güte des Herrn Dr. Höderath vor Kurzem, obgleich ihr Allgemeinbefinden die ganze Zeit über ungestört gewesen, an einer heftigen Entzündung wiederum der Lederhaut auf dem bisher gesunden Auge.

Die Entzündung hatte eine Verdickung des episkleralen Gewebes im oberen Quadranten des Bulbus zur Folge. Herr Dr. Höderath, entschloss sich in Anbetracht der früheren Erkrankung die neugebildete Parthie mit dem Graefe'schen Messer abzutragen und cauterisirte nach sorgfältigster Entfernung alles Krankhaften die ganze Stelle, wo die Neubildung gesessen auf's Ausgiebigste.

Die mir übergebenen Stückchen untersuchte ich mikroskopisch und fand ein aus Granulationszellen zusammengesetztes Gewebe mit spärlichen epithelialen Zellen und vereinzelten Riesenzellen.

Die Untersuchung auf Tuberkelbacillen ergab positiven Befund: es fanden sich in der Nähe einer Riesenzelle zwei Bacillen.

Es handelt sich also auch bei der Erkrankung des bisher gesunden Auges um Tuberculose, die wiederum nicht von der Bindehaut ausging. Es wäre ein merkwürdiger Zufall, wenn anch auf diesem Auge die Tuberkelbacillen, die sich zufällig im 
Bemerkungen zu dem von Schultze mitgetheilten Fall etc. 263

Bindehautsacke aufhielten, die normale Bindehant durchwandert hätten!

Die Erkrankung des bisher gesunden Auges spricht doch eher für eine secundäre Affection, und ich glaube in diesem Umstand eine neue Stütze finden zu dürfen für die Annahme einer secundären tuberculösen Infection in dem von mir publicirten Falle.

Noch ein zweiter Punkt bedarf der Besprechung, und damit komme ich zugleich zu einer Erörterung der Frage, ob unsere bisherigen Kenntnisse über das Wesen der Keratitis parenchymatosa für die Genese dieser Erkrankung im Sinne Schultze's sprechen.

Zum angeblichen Beweise fuir die Richtigkeit seiner Anschauung über die Pathogenese der Keratitis parenchymatosa citirt Schultze S. 152 den von mir erwähnten Befund an der Descemet'sehen Membran folgendermaassen: „- so dass sie auf kurze Strecken manchmal geradezu abgehoben erscheint, aber keineswegs auch nur annähernd so hochgradig, als in dem von Zimmermann beschriebenen Falle".

Und S. 157 heisst es:

"Ausser den Veränderungen der Hornhautsubstanz, die von allen Autoren in derselben Weise, wenn auch in verschiedener Hochgradigkeit, beschrieben wird, erwähnen nun auch Baumgarten, Meier und Zimmermann, dass die Membrana Descemetii mehr oder minder dureh Exsudat abgehoben war, stark welligen Verlauf zeigte, und ebenso wie das Enthothel durch Zellanhäufungen, welche auch noch das erhaltene Endothel überzogen, unterbrochen war. Ich glaube nun, dass gerade diese Befunde für die Genese und Aetiologie der Keratitis parenchymatosa von grösster Wichtigkeit sind n. s. w."

Zu diesem Punkte kann ich nur nochmals betonen, dass, wenn auch die Veränderungen in den hintersten Hornhautschichten die hochgradigsten waren, ich dennoch, gerade wegen den geringen Veränderungen am Endothel und an der Descemet'schen Membran, die in keinem Verhältnisse zu den ausgedehnten Granulationswucherungen der an die Descemetschen Membran angrenzenden Hornhauttheile stehen, mit Berücksichtigung der anderen Momente zu der Ueberzeugung kam, dass die Granulationswucherungen der Hornhaut, das anatomisehe Substrat der parenchymatösen Trübung, in Folge continuirlicher Fortsetzung vom skleraibord aus sich entwickeiten, und nicht durch Dissemination aus der vorderen Kammer zu deuten sind. 
S. 229 heisst es wörtlich:

"Die Membrana Descemetii, welche, wie aus dem Vorhandensein eines eiweissreichen Exsudates hervorgeht, zu Lebzeiten eine, wenn anch geringe Abhebung gezeigt haben muss(!) ist offenbar durch den Härtungsprocess noch weiter abgehoben worden".

Von „Zellanhäufungen, welche auch noch das erhaltene Endothel überzogen", ist in meiner Arbeit nirgends etwas zu lesen. Yan hätte also eher vermuthen dürfen, dass Schultze aus meinen Befunden die gerade entgegengesetzten Schlüsse ziehen würde, als er es wirklich gethan hat.

Auch für den von Schultze mitgetheilten Fall, in welchem die Anamnese einen in frühen Jahren stattgehabten Bluthusten und die objective Untersuchung der Lungen zur Zeit der Hornhautaffection eine geringe Dämpfung der rechten Lungenspitze feststellte, ist es doch weit ungezwungener, eine secundäre Infection des Auges auf dem Wege der Blutbahn anzunehmen, als eine ektogene Infection durch intactes Gewebe, wie es Schultze annehmbar erscheint, ohne dass er auch die geringsten Beweise für einen derartigen Infectionsmodus finden kann! Gegen die am Schlusse seines Nachtrags angeführte Ansicht, dass Erkrankungen des Uvealtractus nichts mit der Entstehung der Hornhauttrübungen zu thun haben, dass sie als reine Nebenbefunde aufzufassen sind, und gegen die Annahme, dass auch bei Grenztumoren der Corneoskleralgrenze resp. des Kammerwinkels die secundären Trübungen der Cornea auf dem Wege durch die vordere Kammer zu Stande kommen, lassen sich verschiedene Bedenken geltend machen:

Vor Allem möchte ich darauf hinweisen, dass die chorioretinitischen Veränderungen, welche man nach Ablauf, öfters aber auch vor dem Eintritt der Keratitis parenchymatosa beobachtet, weit häufiger vorhanden sind, als man frïher annahm.

Hirsehberg ${ }^{1}$ ) gab im Jahre 1888 an, diese Betheiligung des Fundus finde in $16 \%$ statt, im Jahre 1895 nennt er diese Netzhaut- Aderhautveränderungen, wie schon in der v. Hippelschen Arbeit erwähnt wird, "ganz regelmässige" mit dem Bemerken, dass man eben die Fälle länger untersuchen und

1) Hirschberg, „Ueber specifische Hornhautentzündung“, Deutsche med. Wochenschrift, Nr, 25 u. 26. 1888. 
verfolgen müsse, um diese Erkrankungsheerde zu erkennen; v. Hippel $\left.{ }^{1}\right)$ fand dieselben in $60 \%$ der untersuchten Fälle.

Nach diesen Erfahrungen der letzten Jahre hat man doch wohl ein Recht, in diesen ziemlich constant beobachteten Veränderungen der Retina und Chorioidea mehr als zufällige Nebenbefunde zu erblicken, besonders im Hinblick auf die Resultate der Wagenmann'schen ${ }^{2}$ ) experimentellen Untersuchungen über das Auftreten von parenchymatösen Hornhauttrübungen nach Unterbrechung der Blutzufuhr am hinteren Pole des Auges. Die Wagenmann'schen Untersuchungen setze ich als bekannt voraus. Wenn Schultze S. 157 schreibt: "seine Durchschneidungen eines Theiles der Ciliargefässe erzeugten zwar eine graue Trübung der Cornea, dieselbe ging aber entweder bereits nach einigen Tagen zurück, während hochgradige degenerative Veränderungen der Retina eintraten, oder es kam auch bereits nach einigen Tagen zu völliger Nekrose des Bulbus, eine chronische Entzündung der Hornhaut kam nie zu Stande", so hat er offenbar übersehen, dass S. 54 der Wagenmann'schen Arbeit erwähnt ist, dass die Trübung 2-3 Wochen an Intensität zunimmt, dann langsam abblasst, dass sich also der Process nicht nach den Angaben Wagenmann's auf Tage, sondern auf mehrere Wochen erstreckt! Ich selbst habe gelegentlich einer experimentellen Studie über die Narbenverhältnisse des Opticusstumpfes nacl der Resection ${ }^{3}$ ) bei unbeabsichtigt vorgekommenen Durchschneidungen der Gefässe am hinteren Pole beobachten können, dass die parenchymatöse Hornhauttrübung, welche im Anschluss an die Gefässdurchtrennung auftritt, in einigen Fällen nicht nach kurzer Zeit zurückging, sondern, ohne dass das Auge phthisisch wurde, Monate lang bestehen blieb in einem Falle beobachtete ich dass Thier acht Monate lang $^{4}$ ). In Uebereinstimmung mit den Angaben Wagenmann's

1) v. Hippel 1. c. S. 266.

2) Wagenmann, „Experimentelle Untersuchungen über den Einfluss der Circulation in den Netzhaut- und Aderhautgefässen ete." จ. Graefe's Archiv f. Ophthalm. Bd. XXXVI. 4.

3) Zimmermann, "Experimentelle und anatomische Untersuchungen über die Festigkeit der Opticusnarben etc." v. Graefe's Archiv für Ophthalm. Bd. XIII. 2. S. 139.

4) Dieses Thier wurde s. Z. aus äusseren Gründen aus der Versuchsreihe geschaltet und fand deshalb in meiner Arbeit keine Berücksichtigung. 
habe ich die Erfalirung gemacht, dass es bei diesen Durchschneidungen auf die Anzahl und die Grösse der durehschnittenen Gefässe ankommt; in denjenigen Fällen, bei denen es zu einer raschen Rückbildung der Hornhauttrübungen kam, waren eben weniger Blutgefässe verletzt und die Phthisis bulbi tritt ein, "wenn die Grenze dessen, was das Auge verträgt, überschritten wird."

Die chronisch-parenchymatöse Trübung kann auftreten, wenn bei sehr ausgedehnter Gefässdurchschneidung doch noch die Circulation zur Erhaltung des Auges gerade hinreichend bleibt.

Aehnliche Resultate erzielte Koster ${ }^{1}$ ) bei seinen Untersuchungen über die Circulationsverhältnisse im Auge nach Unterbrechung der Abfuhr des Blutes durch die Venae vorticosae.

Wenn ferner Schultze S. 157 schreibt: "Irgend welche Veränderungen der Gefässe des Uvealtractus sind nun aber in den anatomisch untersuchten Fällen von Keratitis parenchymatosa bisher niemals gefunden worden", so bedarf es, um dies richtig zu stellen, nur eines Hinweises auf die anatomische Untersuchung Hennicke's ${ }^{2}$ ) aus der Jenaer Klinik, welcher Veränderungen der Aderhaut bei Keratitis parenchymatosa beschreibt.

S. 135 dieser Publication heisst es wörtlieh: "In den äquatorialen Theilen des Bulbus sieht man ausserdem auch mehrere chorioretinitische Heerde. Die Veränderangen innerhalb desselben erstrecken sich hauptsächlich auf die äusseren Schichten der Retina. An einigen Stellen haben sie bis zur vollständigen Atrophie der Stäbchen- und Zapfenschicht und der äusseren Körnerschicht geführt u. s. w."

Obgleich nun bei der menschlichen Keratitis parenchymatosa Veränderungen im Cireulationsapparat der Uvea nicht constatirt sind, bei welchen wir nach den Beobachtungen der Thierversuche das Auftreten von Keratitis parenchymatosa erwarten dürfen, so ist dennoch diese Möglichkeit der Entstehung auch im menschlichen Auge nicht einfach von der Hand zu weisen. Denn einmal ist das Material ein zu seltenes, so dass nur ganz vereinzelte Fälle zur anatomischen Untersuchung gelangten, und zweitens entstelıt eben die parenchymatöse Hornhauttriibung anch secundär im Anschluss an Erkrankungen resp. Neubildungen der directen Umgebung-der Hornhaut, so dass hier wieder andere veranlassende

1) Koster, v. Graefe's Archiv f. Ophthalm. XLI. 2. S. 30 ff.

2) Hennicke, Ueber Keratitis parenchymatosa bei Bären, v. Zehender's Klinische Monatsblätter f. Augenheilk. XXXII. Jahrg. 
Momente in Betracht zu ziehen sind. Ich denke hierbei in erster Linie an die sogenannten secundären Formen der Hornhauterkrankung im Sinne Michel's, welche sich klinisch manchmal längere Zeit, oder wie in dem von v. Hippel ${ }^{1}$ ) untersuchten Falle überhaupt als primäre Form dargestellt haben.

Michel nimmt bekanntlich für die Entstehung mancher Formen von Keratitis parenchymatosa eine luetische Affection der Gefässe des Randschlingennetzes an. Dass Ernährungsstörungen in der nächsten Umgebung der Hornhaut zu correspondirenden parenchymatösen Trübungen führen können, ist ja schon längere Zeit erwiesen.

Erinnern möchte ich hierbei auch an die Versuche Michel's'), welchem es gelang, durch Injection einer indifferenten Flüssigkeit in der Umgebung der Cornea, in dieser selbst eine sich vom Rande her nach der Hornhantmitte vorschiebende Parenchymtrübung zu erzielen.

Durch diese experimentellen Untersuchungeu wurde das Verständniss für die Vorgänge beim Zustandekommen mancher Formen von parenchymatösen Trübungen sehr gefördert. Es scheint nach den bisherigen klinischen Beobachtungen und nach den Erfahrungen, welche dureh die experimentellen Studien gewonnen sind, keinem $Z_{w}$ eifel zu unterliegen, dass der Modus der Genese der parenchymatösen Trübungen bei Erkrankungen in der Umgebung der Hornhaut (resp. Tumoren der Sklera, des Kammerwinkels ete.) nicht den Schultze'schen Annahmen entspricht, dass also nichit erst eine Resorption von toxinhaltigem Kammerwasser in die hinteren Lamellen des Hornbantgewebes nach einer toxischen Läsion des Endothels erfolgen muss.

Hiergegen spricht unter anderen auch die Beobachtung, dass die anfänglichen und hauptsächlichen Trübungsheerde meist in der unmittelbaren Nähe des Erkrankungsheerdes liegen, und nicht eine gleichmässige, mehr oder minder diffuse Trübung: (zuerst in den hintersten Schichten) bei solchen Fällen aufzutreten pflegt, wie es doch im Sinne der Schultze'sehen Voraussetzungen wahrscheinlich sein würde.

Bei einigen Versuchen auf experimentellem Wege beim Kaninchen parenchymatöse Hornhautentzündung hervorzurufen, erhielt ich in zwei Fällen nach Impfung von Tuberkelbacillen-

1) v. Hippel, „Ueber Keratitis parenchymatosa", v. Graefe's Archiv f. Ophthalm. Bd. XXXIX. 3. S. $204 \mathrm{ff}$.

2) Michel, Lehrbuch d. Augenheilk. II. Aufl. S. 234.

v. Graefe's Archiv für Ophthalmologie. XuIV. 
reincultur in die Sklera in der unmittelbaren Nähe der in den vorderen Bulbusabschnitt eintretenden Arterie eine circumscripte parenchymatöse Trübung der Hornhaut, welche der geimpften Stelle in der Höhe entsprach; dieselbe ging von dem Limbus aus in einer dem Heerd in der Sklera ungefähr entsprechenden Höhenausdehnung und ragte als gesättigte gleichmässige Trübung in die Hornhant ca. 2-3 mm hinein. Da die tuberculöse Neubildung, rasch wuchernd nach dem Glaskörperranme perforirte, musste ich das Auge, um einen reinen Befund zu haben, enucleiren. Die anatomische Untersuchung zeigte als Ursache der Hornhauttrübung ein Granulationsgewebe mit neugebildeten Gefässen, das sich direct in dem skleralen Granulationsheerde fortsetzte. Ein Zusammenhang mit den Gefässen war nicht mehr zu erkennen, da das ganze ursprïngliche Gewebe in der Neubildung aufgenommen war. In den übrigen Theilen, abgesehen von der allernächsten Umgebung der Trübungszone, bot die Hornhaut nahezu normale Verhältnisse.

Einen analogen Befund bot mir vor Kurzem die anatomische Untersuchung eines mir von auswärts zur Untersuchung zugesandten menschlichen Bulbus. Der Fall stammt aus der Klinik des Herrn Dr. Höderath und war unter der Diagnose eines Tumors der Corneoskleralgrenze im Endstadium in Behandlung gekommen.

Ich fand eine ca. bohnengrosse, von der oberen Corneoskleralgrenze seitlich bis nahe an den Aequator und nach hinten in den Glaskörper vorgedrungene tuberculöse Neubildung, welche wahrscheinlich vom Ciliarkörper ausging; die Iris ist um das 3-4 fache verdickt, so dass die vordere Kammer sehr seicht erscheint. Die Hornhaut zeigt die für parenchymatöse Processe charakteristischen Substrate: Rundzellenanhäufungen aber nur in kleineren Plaques, Gefässneubildung und Veränderung der Fibrillen in Zusammenhang und Gestalt nebst Vermehrung der Stromakerne. Diese Veränderungen sind am intensivsten in der Nähe des Tumors und zwar in den mittleren Hornhautschichten und nehmen gegen die centralen Theile der Cornea und gegen die der Descemeti'schen Membran benachbarten Schichten hin ab. Irgend welche grössere Zellanhäufungen in der Nähe der Descemeti'schen Membran sind nicht vorhanden.

Trotzdem in diesem Falle bei der starken Mitbetheiligung der Regenbogenhaut mehr denn in anderen Fällen, wenn die Schultze'sche Auffassung zu Recht bestünde, Veränderungen der Descemeti'schen Membran und ihres Endothels, resp. eine stärkere 
Betheiligung des Hornhautgewebes in den der Descemeti'schen Membran anliegenden Schichten der Cornea zu erwarten gewesen wären, finden sich, wie erwähnt, die erheblicheren Veränderungen in den an den Tumor angrenzenden Bezirken und zwar in den vorderen Schichten.

Weiter gegen das Centrum der Cornea zu, sehen wir noch leichte Durchsetzung mit Rundzellen und Aufloekerung der fibrillären Elemente neben Kernvermehrung.

Aehnliche Fälle liessen sich wohl noch mehrere anführen, wenigstens zeigt uns die Beobachtung von klinischen Fällen nicht gerade selten das Auftreten von parenchymatösen Trübungen der Cornea, welche sicher ohne Vermittlung des Kammerinhaltes zu Stande kommen.

Ich möchte noch kurz einen hierher gehörigen Krankheitsfall erwähnen, den ich in Jena beobachtete und der, wie mir scheint, besonders für diese Anschauung spricht. Es handelte sich um ein sehr progressiv verlaufendes, aus einer rasch eitrig eingeschmolzenen kleinen Pustel des episkleralen Gewebes hervorgegangenes Geschwür, welches ea. $3 \mathrm{~mm}$ nach innen unten vom inneren unteren Hornhautrande sass. Trotz der Cauterisation und der üblichen stark antiseptischen Behandlung (Pinselung des Geschwürsgrundes und der steilfallenden, kraterförmigen Wände mit Sublimat 1:100,0!) nahm das Uleus rapid an Ausdehnung zu, so dass es bald die Grösse und den Umfang einer halben Erbse erreicht hatte. Zu einer Zeit, als schon dichte Glaskörpertrübungen den drohenden Durchbruch nach innen verriethen (am vierten bis fünften Tage) trat eine rasch zunehmende, vom Rande der Hornhaut in der unmittelbaren Umgebung des Uleus beginnende parenchymatöse Trübung der Cornea auf, welche sich in einer Höhenausdehnung von ea. $4 \mathrm{~mm}$ nach dem Centrum der Cornea vorschob, ohne jedoeh dieses zu erreichen. Die übrigen Parthieen der Hornhaut blieben klar. Als es noch im letzten Momente gelang, nach ausgiebigster, bis in's Gesunde hinein ausgeführten Cauterisation das Geschwür zum Stillstand zu bringen, bildete sich im gleichen Schritte, wie die Heilung: des Ulcus fortschritt, die Trübung zurück, so dass eine vollständige Aufhellung erfolgte.

Obgleich hier schon eine starke Mitbetheiligung des hinteren Augenabschnittes aufgetreten war, also doch wohl auch in das Kammerwasser Toxine eingetreten sein dürften - wenn dies überhaupt in Betracht gezogen werden soll - also die denkbar günstigste Gelegenheit zar Eutstehung einer Keratitis parenchy- 
matosa im Sinne Schultze's gegeben war, trat dennoch jene Form von parenchymatöser Trübung auf, welche sicherlich nur zu Stande kommt durch ein directes, unmittelbares Uebergreifen des krankhaften Processes selbst auf das Gewebe der Cornea, oder aber durch eine intensive Ernährungsstörung irgend welcher Art, wie sie durch die Nähe des Erkrankungsheerdes direct verursacht wird.

Ich glaube, die angef ührten Beispiele dürften genügen, um zu zeigen, dass die Ansicht Schultze's über das Zustandekommen der Keratitis parenchymatosa sich weniger stützen kann auf unsere bisherigen klinischen und anatomischen Erfahrungen,- als die Ansicht derer, welche, wie Wagenmann, einer Erkrankung von Blutgefässen, resp. der in ihrem Gefolge auftretenden Ernährungsstörungen in der Genese der parenchymatösen Hornhauttrübung eine hervorragende Bedeutung zuschreiben.

Dass bei chronisch veränderter Beschaffenheit des vorderen Kammerinhaltes auch obne directe traumatische Läsion des Hornhautendothels parenchymatöse Hornhauttrübungen auftreten können, habe ich selbst offters beobachtet, als ich, um das Endothel nicht zu verletzen, die Injectionen von Sublimat in die vordere Kammer von der Sklera aus durch die Iriswurzel ausführte. Es gelingt bei einiger Uebung, besonders wenn man nur einen Theil des Kammerinhaltes aspirirt, die Einspritzungen zu vollenden, ohne dass man die hintere Hornhautfläche berührt. Stärkerer Druck ist zu vermeiden, da man sonst gleichfalls parenchymatöse Trübungen erhält, die durch den Druck verursacht sind.

Diese diffusen Trübungen, die sich bei regelrecht ausgef ührter Infection im Verlaufe einiger Stunden ausbildeten, gingen manchmal zurück im Verlaufe einiger Tage bis Wochen, in einigen Fällen sah ich jedoch ein Fortbestehen derselben durch mehrere Monate hindurch. Es ist anzunehmen, dass diese Trübungen dureh Einwirkung des Sublimats aus der vorderen Kammer zu Stande kamen.

Entsprechend dem klinisehen Bilde zeigte der anatomische Befund die stärksten Veränderungen der Hornhautfibrillen (Quellung und Lockerung der Fibrillen bei leicht welligem Verlaufe, Vermehrung der Stromakerne; nur in Fällen, in denen sehr starke Sublimatlösung injieirt war, war eine Durehsetzung mit Rundzellen in Hornhaut, vorderer Kammer und Iris nachweisbar).

$\mathrm{Ob}$ den Toxinen eine ähnliche Einwirkung auf das Hornhautendothel zukommt, wie den chemischen Giften, ist vorerst noch nicht bewiesen, ebenso wenig, wie wir überhaupt wissen, 
Bemerkungen zu dem von Schultze mitgetheilten Fall etc. 271

ob und in welcher Quantität sie bei tuberculösen Erkrankungen im vorderen Kammerinhalte vorhanden sind.

Aber selbst vorausgesetzt, dass ihnen eine ähnliche Wirkung, wie den chemischen Giften eigenthümlich ist, dürften wir doch wohl nach Analogie der Sublimattrübung eher eine gleichmässige, diffuse, von hinten nach vorne fortschreitende Trübung der Cornea erwarten, nicht aber solche Formen von Parenehymtrübungen, die von der Umgebung der erkrankten Stelle vom Hornhautrande ausgehen. Auch bei constitutionellen Leiden, z. B. bei der Lues, pflegt die Keratitis parenchymatosa am Limbus zu beginnen.

Ich luatte in den letzten Wochen Gelegenheit, mehrere, an ausgesprochener Keratitis parenchymatosa erkrankte Thieraugen zu untersuchen, über deren Befund an anderer Stelle ausführlich berichtet werden soll.

In den bisher gewonnenen Resultaten erblicke ich gleichfalls keine Stütze für die Schultze'sche Ansicht, wohl aber sprechen gewisse Umstände dafür, dass dem Blutgefässapparat wenigstens in einem der Fälle eine grosse Bedeutung an dem Zustandekommen der parenchymatösen Hornhanttrübung zuzuschreiben ist. Die Section des an typischer Keratitis parenchymatosa (gleichmässige, diffuse Trübung der Hornhaut beider Augen) beiderseits exblindeten Axishirsches hatte eine allgemeine Gefässerkrankung (Endarteriitis und Mesarteriitis) ergeben, an deren Folgen das Thier zu Grunde ging.

Da Herr Sanitätsrath Königshöfer sich die ausführliche Beschreibung dieses interessanten Falles vorbehalten hat, bin ich nicht in der Lage, hier auf den näheren Befund der Angen einzugehen. Eines jedoch möchte ich erwähnen: dass die arteriellen Gefässe in der Umgebung der Cornea und im vorderen Abschnitte der Gefässhaut so hochgradig verändert sind, dass stellenweiser Gefässverschluss durch Wucherung der Intima zu constatiren ist.

Jedenfalls erblicke ich in diesem Falle eine grosse Stütze der Wagenmann'schen Auffassung über die Genese der Keratitis parenchymatosa. 